\title{
How do heterogametic females survive without gene dosage compensation?
}

\author{
H SHARAT CHANDRA \\ Department of Microbiology and Cell Biology, Indian Institute of Science, \\ Bangalore 560012 , and \\ Centre for Cellular and Molecular Biology, Hyderabad 500007 , India
}

MS received 22 March 1991

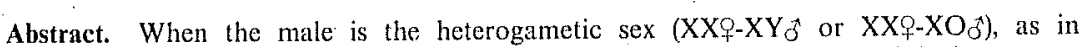
Drosophila, orthopteran insects, mammals and Caenorhabditis elegans, X-linked genes are subject to dosage compensation: the single $\mathrm{X}$ in the male is functionally equivalent to the two $\mathrm{Xs}$ in the female. However, when the female is heterogametic ( $\mathrm{ZZ} \delta$-ZWO), as in birds, butterllies and moths, Z-linked genes are apparently not dosage-compensated. This difference between $X$-linked and $Z$-linked genes raises fundamental questions about the role of dosage compensation. It is argued that (i) genes which require dosage compensation are primarily those that control morphogenesis and the prospective body plan; (ii) the products of these genes are required in disomic doses especially during oogenesis and early embryonic development; (iii) heterogametic females synthesize and store during oogenesis itself morphogenetically essential gene products - including those encoded by Z-linked genes - in large quantities; (iv) the abundance of these gene products in the egg and their persistence relatively late into embryogenesis enables heterogametic females to overcome the monosomic state of the $\mathrm{Z}$ chromosome in $\mathrm{ZW}$ embryos. Female heterogamety is predominant in birds, reptiles and amphibians, all of which have megalecithal eggs containing several thousand times more maternal RNA and other maternal messages than eggs of mammals, Caenorhabditis elegans, or Drosophila. This increase in egg size, yolk content and, concomitantly, the size of the maternal legacy to the embryo, may have facilitated female heterogamety and the absence of dosage compensation.
\end{abstract}

Keywords. $Z$ chromosomes; dominant maternal genes; megalecithal eggs; maternal mRNA.

\section{Introduction}

Dosage compensation is a classic example of the precision of genetic adaptation (Muller 1948). In Drosophila melanogaste it is believed that the evolution of dosage compensation has enabled the male to survive the deleterious effects of the monosomic (single-copy) condition of the $X$ chromosome. Monosomy for the two other large chromosomes - which are autosomes, and lack such compensatory mechanisms - are lethal. However, analysis of the dose requirements for normal development of different segments of the Drosophila genome suggests that the number of dose-sensitive genes is not large. Heterozygous deletions or duplications of most single loci have little or no obvious effect on development. In a study of the effects of segmental aneuploidy, it was estimated that the number of dose-sensitive genes in the Drosophila genome is at least fifty seven (Lindsley et al. 1972). One locus causes lethality when present in triplicate in an otherwise diploid genetic background. Whereas subsequent analyses have revealed additional dose-sensitive genes, it is unlikely that their number would go beyond a few hundred. Thus, the imbalance observed in monosomies (and 
trisomies) is probably attributable to the cumulative effects of a number of dosesensitive genes rather than to any unknown property of whole chrosomosomes (John and Miklos 1988).

One result of dosage compensation is that the sexual dimorphism in phenotype, which might develop if dosage compensation did not occur, is avoided. Clearly, however, at least some X-linked genes - those concerned with sex determination would not be subject to dosage compensation (in the usual phenotype sense), because what such genes presumably require is a dosage effect rather than compensation (see, for example, Chandra 1986). Many other X-linked - such as those coding for enzymes - are subject to dosage compensation even though they appear to be 'dose-insensitive'; drastic changes in the levels of their products do not lead to clear-cut fitness effects (Kacser and Burns 1981).

\section{Evolution of dosage compensation}

An early step in the evolution of heteromorphic sex chromosomes is believed to be a gross reduction in or elimination of recombination between the "primitive" $X$ and $Y$ chromosomes. Suppression of recombination would lead to the isolation of the male-determining gene or genes in the primitive $Y$ chromosome. Non-functional alleles resulting from mutations would accumulate in the $\mathrm{Y}$ because, in the absence of recombination, a functional allele at the corresponding locus on the $\mathrm{X}$ would not be able to crossover to the $Y$ (Charlesworth 1978). In a population of $Y$ chromosomes, then, there would be some with zero and others with one, two, or more non-functional alleles. If population size is small, there is a non-zero probability with which each of these chromosomes would be lost by chance alone. When a $Y$ chromosome that is free of non-functional mutations is lost, there would be no mechanism by which another mutation-free (wild-type) $\mathrm{Y}$ can be generated because of the absence of recombination. This principle, referred to as Muller's ratchet, would repeatedly eliminate that class of Y's with the lowest number of mutations. As a consequence, $Y$ chromosomes with non-functional genes would accumulate in the population. Selection would then favour dosage compensation of those $X$-linked genes which now exist in single copy in the male. Enhancement of transcription of $\mathrm{X}$-linked genes would be expected to be nonspecific to some extent (Charlesworth 1978). However, it is not clear whether dosage compensation evolves at the level of large chromosomal domains or on a gene-by-gene basis.

Rice (1987) has suggested genetic "hitchhiking" as another mechanism by which the reduced genetic activity of the $\mathrm{Y}$ chromosome could evolve following supression of recombination. This mechanism is mediated by the fixation of Y-linked mutations that are linked to other, beneficial genes. There would be concurrent evolution of dosage compensation as in Charlesworth's model. Hitchhiking can reinforce Muller's ratchet as well as operate in conditions in which the ratchet is ineffective. An attractive aspect of this model is that it appears to permit either the evolution of dosage compensation or its absence ("dose tolerance"). In case of dose tolerance, the two sexes would have the capacity to accommodate different concentrations of $\mathrm{X}$ - and Z-linked gene products.

Dosage compensation has also been demonstrated in orthopteran insects (XX. XO) (Hebbert 1984), the nematode Caenorhabditis elegans (Meyer 1988), and in 
mammals, in which it is a result of inactivation of one of the two X chromosomes in the female (Lyon 1961).

Dosage compensation may not be necessary when the number and morphology of chromosomes is the same in the two sexes. But it appears that dosage compensation is not an absolute requirement even for organisms with distinctly heteromorphic sex chromosomes. For example, in birds and butterflies ( $\mathrm{ZZ}{ }_{0}^{*}-\mathrm{ZW}$ ), a large body of evidence suggests that there is, instead of compensation, a dosage effect of Z-linked genes (Stehr 1959; Cock 1964; Johnson and Turner 1979; Baverstock et al. 1982). Genes coding for the enzyme 6phosphogluconate dehydrogenase in butterflies (Johnson and Turner 1979), and aconitase $I$ in birds (Baverstock et al. 1982) are Z-linked, and both show a clear dosage effect. Data on several sex-linked morphological characteristics in birds are consistent with a dosage effect of Z-linked genes (Cock 1964). Moreover, the $Z$ chromosome does not show cytological indications of dosage compensation (such as enlargement of the single $X$ in male Drosophila or $X$-chromatin in female mammals). In the moth Choristoneura, genes controlling hemolymph colour are not dosage compensated (Stehr 1959). Among butterflies, female-limitation of colour polymorphism, mimicry and certain non-mimetic polymorphisms are thought to result from an interaction between autosomal genes and uncompensated Z-linked genes (Stehr 1959; Sheppard 1961; Cock 1964; Grula and Taylor 1980). A large proportion of the genes controlling female mate-selection behaviour and male courtship signals are located on the $Z$ chromosome (Grula and Taylor 1980), and genetic data on these phenotypes are consistent with the view that most, if not the whole, of the $\mathrm{Z}$ chromosome lacks dosage compensation (Johnson and Turner 1979; Grula and Taylor 1980). Taken together, these results suggest that many organisms are fully viable in spite of a two-fold disparity in the functional levels of Z-linked genes.

Among insects, dosage compensation occurs among the Orthoptera and Diptera, but not among the Lepidoptera, and this has led Hebbert (1984) to suggest that dosage compensation was present in related archeopteran ancestors of these groups and that it has been secondarily lost in the Lepidoptera. It has been noted that in species in which dosage compensation has been shown to occur (Drosophila, orthopteran insects, mammals and Caenorhabditis elegans), the males are heterogametic, whereas in groups lacking dosage compensation (birds, moths and butterflies), females are heterogametic (Cock 1964; Johnson and Turner 1979; Baverstock et al. 1982). The $\mathrm{Z}$ chromosome, like the $\mathrm{X}$, is invariably one of the larger chromosomes and constitutes a significant part of the haploid genome (Ohno 1987). In birds the $Z$ chromosome is of the same size as the $X$ chromosome of mammals (Ohno 1987). Therefore, the viability of heterogametic females in spite of an absence of dosage compensation raises questions about the role of dosage compensation. This problem has acquired additional interest in the light of recent results on butterflies which suggest that the absence of dosage compensation also may be the result of a precise adaptation (Stehr 1959; Grula and Taylor 1980). In the remainder of this paper I consider a possible explanation for the viability of heterogametic females in spite of an absence of dosage compensation. Our starting point is the observation, first made by Lyon (1974, page 259), that there is an apparent difference in egg size, egg content and timing of zygotic gene activity between male-heterogametic and female-heterogametic organisms. 


\section{Egg size and content in relation to female heterogamety}

Female heterogamety occurs in a majority of amphibian species, including Xenopus laevis (Bull 1983; Schmid 1983; Ohno 1987) [although heteromorphic sex chromosomes have apparently been observed in the females of only one strain of this species (Ohno 1987)]. X. laevis is the best characterized amphibian in terms of its oogenesis and early development and can be considered as representative of amphibians generally. The Xenopus egg is about 4000 times the size of the mouse or human egg and contains roughly 10,000 times as much stored mRNA (Wilkins 1986; Tata 1986). The same relationship is thought to apply to proteins, lipids and other egg constituents. The C. elegans egg $(40 \mu \mathrm{m} \times 50 \mu \mathrm{m})$ is even smaller than the mouse egg and it may be the smallest egg so far studied. All birds studied to date show female heterogamety, and the so-called yolk in the avian egg represents a giant oocyte whose diameter can reach a few centimetres. However, irrespective of whether the organism is oviparous or viviparous, vertebrate or invertebrate, the amount of RNA per unit mass of the mature egg, as well as the complexity of this RNA, are constant (Wilkins 1986; Tata 1986).

\subsection{Sex chromosome monosomy and oogenesis}

An unexpected result of molecular studies of embryogenesis in Drosophila using cDNA libraries is that over $80 \%$ of the genes expressed as late as in the gastrula are also expressed in the oocyte, suggesting that a significant fraction of the maternal genome is active in oogenesis (Wilkins 1986; Davidson 1986). Such results are consistent with evidence obtained from embryos as diverse as those of Drosophila, Caenorhabditis, sea urchin, Xenopus and mammals, that there is a quantitative dominance of maternal transcripts in the zygote and during the immediate postzygotic development (Wilkins 1986; Davidson 1986). Maternal control of some early embryonic events may be obligatory because normal embryogenesis appears to require a diploid dosage of a number of maternal genes during the preceding oogenesis (Wilkins 1986). A function of reactivation of the inactive $\mathrm{X}$ in oogonia of human females is thought to be restoration, in effect, of a disomic dosage of $X$ linked genes in the germ line (Gartler et al. 1975). When the XX constitution is female, then this apparent need for diploid dosage of maternal genes is satisfied. On the other hand, at the time of evolution of ZW females the seemingly inadequate gene dosage in the female germline has to be overcome. There are essentially three ways in which this could have come about: increased synthesis and storage of the products of maternally acting genes; a shift in the timing of action of zygotic genes such that they became, in effect. "maternal" genes; and, by a similar shift in the time of action of genes which are both maternal and zygotic, such that they became exclusively maternal genes. In other words, if the pattern of oogenesis, egg size, amount of stored maternal mRNA in the egg, and the time of onset of zygotic gene expression differ sufficiently between representative organisms with female heterogamety and those with male heterogamety, such differences might account for the survival without dosage compensation of the former. If such increased storage of maternal products and shifts in timing of action of developmentally significant genes have in fact occurred, it may be possible to detect such instances by appropriate comparisons between male-heterogametic and female-heterogametic systems. 


\section{Timing of zygotic gene activity}

If the timing of zygotic gene function depends on the extent of stored maternal messages in the egg, one might expect to see a delayed onset of zygotic activity in $X$ enopus relative to that in D. melanogaster, C. elegans or mammals. This appears to be the case. For instance, in the oocytes of Xenopus and other amphibia, large quantities of ribosomes are formed by amplification of ribosomal genes and these maternal ribosomes are stored and used until gastrulation when embryonic cells then numbering about 10,000 - begin transcription (Tata 1986). In contrast, mouse and human eggs store very few ribsomes and transcription begins in the mouse embryo as early as in the 2- to 4- cell stage (Flach et al. 1982) and in the human embryo, between the 4- to 8- cell stages (Braude et al. 1988). Moreover, in mouse, matemal mRNA is actively degraded at the 2-cell stage and by early blastocyst stage the mRNA pool is virtually all zygotic in origin (Davidson 1986), suggesting that embryogenesis need be under the control of stored maternal messages only until then. In neither mammalian species is there evidence of amplification of ribosomal or other genes (Tata 1986). In D. melanogaster zygotic gene expression begins at the blastoderm stage; in $C$. elegans the latest period of onset of expression of the zygotic genome is 90- to 125-cell stage (Wilkins 1986). Mouse embryos treated with actinomycin-D do not develop beyond first cleavage whereas Xenopus embryos similarly treated (or enucleated) continue to develop until the 4000-cell blastula stage (Tata 1986; Davidson 1986).

Among the approximately 50 genes determining metameric pattern in Drosophila, 6 function both maternally and zygotically (Akam 1987; NussleinVolhard et al. 1987). For a few others, such as hopscotch and dissheveled, either maternal or zygotic function is sufficient for normal segmentation to occur (Nusslein-Volhard et al. 1987). If a Drosophila pattern gene acts zygotically or later in development, one might expect the Xenopus 'homolog' to be maternally acting. Such an expectation seems to be met in the case of $X I H$ box 2, a maternally expressed homeobox-containing locus in $X$. laevis which shares sequence homology with caudal, a Drosophila pattern gene (Wright et al. 1987). XIH box 2 and caudal are among the first such genes for which comparative data are available. Whereas caudal is expressed maternally as well as during embryogenesis, XIH box 2 is expressed almost entirely in stage 2 and 3 oocytes when many gene products are stockpiled in the oocyte for utilization during early development (Wright et al. 1987).

\section{Dose-sensitivity of genes during early development}

Genes performing 'housekeeping' functions would be required to act throughout the life of an organism, and many such genes appear to be dose-insensitive, or largely so. For example, the copy number of genes coding for enzymes is not a significant factor in the control of metabolic flux (Kacser and Burns 1981). Similarly, heterozygotes for human metabolic errors are not readily distinguishable from normal homozygotes because of a large overlap in enzyme levels. Such experience, and the results of studies on the evolution of dominance, have led to the view that because of the structure of enzyme networks and the interactions among enzymes, their substrates, and products, "a 50\% reduction in activity, a common feature for many mutants, is not detectable in the phenotype" (Kacser and Burns 1981). 
In contrast to housekeeping genes, the products of many genes performing morphogenetic functions would be expected to be required exclusively or primarily during early embryonic development. It appears, also in seeming contrast to housekeeping genes, that several genes affecting body plan and pattern are dosesensitive in the appropriate genetic background. For instance, in Drosophila, while elimination of runt ${ }^{+}$, an X-linked gene, causes periodic deletions in the pattern of segmentation, an increased runt ${ }^{+}$dosage causes an "anti-runt" phenotype (Gergen and Wieschaus 1986). Controlling the level of runt activity is a critical aspect of positional identity determination in blastoderm cells (Struhl 1983). A maternal homeotic gene, extra sex combs ${ }^{+}\left(\operatorname{esc}^{+}\right)$, interacts with other pattern genes in a dosedependent manner (Struhl 1983). Dose-dependent relationships are also seen between the maternal anterior gradient product of the bicoid (bcd) gene and its target, hunchback (hb) (Driever and Nusslein-Volhard 1989; Struhl et al. 1989). The pattern of expression of $h b$ embryos derived from mothers carrying different numbers of copies of $b c d$ shows that $h b$ gene activation is sensitive to the concentration of bed protein such that 2 -fold or perhaps even smaller differences in concentration of bed protein are sufficient to trigger the activation of $h b$ in an allor-none fashion (Struhl et al. 1989). Other, subordinate control genes also appear to respond to the concentration-dependent action of this morphogen (Driever and Nusslein-Volhard 1989 or Struhl et al. 1989). In the gene torso (tor), which is required for terminal segmental development, gain-of-function lesions lead to a distinct phenotype that can be rescued by loss-of-function mutations in the downstream gene tailless (Klingler et al. 1988; Strecker et al. 1989). Gene dosage effects are also seen in the processes which lay down the dorsoventral pattern (Ingham 1988). In general, the graded phenotypes of maternal mutants and the dosedependent activation of down-stream genes by maternal gradients (Manseau and Schupbach 1989) reflect the importance of gene and product dosage in early patterning of the Drosophila embryo. These results do not imply that dose relationships are not important subsequently, but it appears that most genes showing dose-dependent effects later in development contribute to multicomponent structures such as ribosomes (Kongsuwan et al. 1985), spliceosomes (Last et al. 1987), the organized cytoskeleton (Regan and Fuller 1988) and the muscle (Homyk and Emerson 1988) in which stoichiometric ratios may be important. For example, many Minute mutations in Drosophila lead to growth retardation because the ribosomes are defective (Kongsuwan et al. 1985).

\section{Dominant maternal genes affecting body pattern}

The hypothesis outlined here would lead to two predictions: (a) to the extent that sex-linked genes are involved in primary patterning, dosage compensation mutants ought to show pattern defects, and (b) loss-of-function mutations affecting pattern will behave as dominants. Relevant to the first prediction is the observation that in $D$.

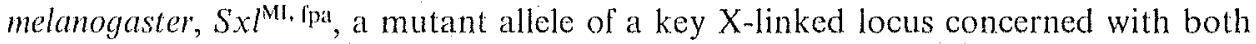
sex determination and dosage compensation, leads to ventral-to-dorsal homeotic transformation in males (Maine et al. 1985). There are several mutations among Drosophila pattern genes which appear to meet the expectations of (b), above. Bicaudal, is a dominant maternal gene (Mohler and Wieschaus 1986), and reduction of Bicaudal ${ }^{+}$gene product by a factor of two or more reduces or abolishes the 
normal anterior-posterior polarity of the embryo and anterior structures are replaced by posterior stuctures (Manseau and Schupbach 1989). Three dominant mutations in the X-linked gene Ovo, which acts zygotically and may also act maternally, exhibit defects in oogenesis which lead to sterility (Mohler and Wieschaus 1986). Dicephalic, a semi-dominant maternal-effect autosomal mutation, alters the anterior-posterior embryonic patterning (Lohs-Schardin 1982). Another maternal gene, dorsal, also has a semi-dominant phenotype in heterozygotes (Busson et al. 1983). Mutations in fushi tarazu ( $f t z)$, a zygotic gene, lead to pattern deletions. The pattern of expression of $f t z$ is controlled by an X-linked maternal effect gene, $f s(1) N^{211}$, demonstrating that a maternal-effect mutation can alter the spatial distribution of a zygotic gene product involved in the segmental patterning of the embryo (Anderson and Nusslein-Volhard 1984). As noted previously, mutations in tailless act as dominant maternal suppressors of a hyperactive allele of torso, a zygotic gene, suggesting that the torso product acts in a dose-sensitive manner, through or in concert with the tailless product (Klingler et al. 1988).

The possibility that some pattern genes which appear dose-insensitive now evolved under conditions in which their own dose or that of other genes with which they interact was important is suggested by the observation that loss-of-function mutations such as $d a$ and sis-a are recessive only so long as the females have a wildtype dose of $S x l^{+}$alleles (Cline 1986) and by the data on esc cited previously. The esc ${ }^{-}$ phenotype depends on the number of copies of Polycomb $(P c)$ and the intensity of phenotypic change among esc $-/ e^{-} c^{-}$progeny of $e \mathrm{sc}^{-} / \mathrm{esc}^{-}$mothers depends on the number of copies of bithorax (Struhl 1983). Similarly, cellular identity during Drosophila segmentation depends not only on the activity of Ultrabithorax ${ }^{+}$ $\left(U b x^{+}\right)$but also on its concentration. The interaction between $U b x$ and the Antennapedia complex in the development of segment identity is dose-sensitive only when the embryos carry one copy of $P c$ (Smolik-Utlaut 1990). These effects of $P c$, $\mathrm{ft} z$ and runt may be explicable in terms of a model, suggested by Tartof and associates (Locke et al. 1988), for the action of certain dominant modifiers of position-effect variegation.

One reason for the relatively small number of dominant maternal pattern genes known so far may be that this number is in fact small in D. melanogaster. Another reason may be that very few systematic searches have been carried out for such genes. In one such search, five regions of the $\mathrm{X}$ chromosome of D. melanogaster were identified, each showing specific dominant interaction with the zygotic gene Kruppel (Tricoire 1988). Two of these regions also showed dominant interactions with either hunchback or hairy (Tricoire 1988).

In $C$. elegans, a screen for dominant, temperature-sensitive maternal-effect embryonic lethals yielded eight mutations at six loci. Mutations at three of these loci result in only maternal effects whereas those at the remaining three cause both maternal and zygotic effects. One of the mutations may be a loss-of-function allele at a haplo-insufficient locus while another may be a gain-of-function "poison" mutation (Mains et al. 1990).

\section{Conclusions}

Thus it appears that in cortain female-heterogametic species a type of non-specific and large increase in the quantity of maternal messages occurs during oogenesis 
and egg maturation. If these messages persist relatively late into embryogenesis, at least until morphogenetic commitments are made, they may provide necessary and sufficient conditions to overcome the monosomic state of the $\mathrm{Z}$ chromosome in $\mathrm{ZW}$ embryos. Increased synthesis and storage of particular maternal products may be facilitated by a general increase in egg size and ovipary, but increased egg size may evolve for reasons unrelated to dosage compensation (Sargent et al. 1987). In Drosophila, the total number of genes affecting the informational content of the egg for embryonic pattern formation is probably no larger than fifty (Akam 1987; Nusslein-Volhard et al. 1987). If the number of dose sensitive genes among them is small, then transition from male heterogamety to female heterogamety might occur even without a significant increase in egg size because the amount of "extra" maternal RNA needed to permit such a shift might not be large. If these arguments are valid, it would mean that the absence of dosage compensation may come about by an increase in the maternal, zygotic or early embryonic levels of the products of a small number of dose-sensitive genes without this being necessarily reflected in an increase in egg size. This would mean that while large egg size might facilitate shifts from male heterogamety to female heterogamety, it may not be a precondition for such shifts. For this reason, it is not clear whether comparison of egg size with type of heterogamety, say among closely related insects, would provide an adequate test of this hypothesis. A more appropriate test would appear to be comparison of the amount of maternal mRNAs of pattern genes in the egg, the timing of activity of such genes, and whether loss-of-function mutations in such pattern genes behave as dominants.

\section{Acknowledgements}

I thank Dr. Stanley Gartler for posing this question to me and for stimulating discussion, Drs P. Babu, Vani Brahmachari, R. Gadagkar, N. V. Joshi, K. Vijay Raghavan and, especially, V. Nanjundiah for helpful comments. This work was supported in part by the Jawaharlal Nehru Centre for Advanced Scientific Research, Bangalore and the Department of Biotechnology, Government of India.

\section{References}

Akam M. 1987 The molecular basis for metameric pattern in the Drosophila embryo. Development 101: $1-22$

Anderson K. V. and Nusslein-Volhard C. 1984 Information for the dorsal-ventral pattern of the Drosophila embryo is stored as maternal mRNA. Nature (London) 311: 223-227

Baverstock P. R., Adams M., Polkinghome R. W. and Gelder M. 1982 A sex-linked enzyme in birds Z-chromosome conservation but no dosage compensation. Nature (London) 296: 763-766

Braude P., Bolton V. and Moore S. 1988 Human gene expression first occurs between the four- and eight-cell stages of preimplantation development. Nature (London) 332:459-461

Bull J. J. 1983 Evolution of sex-determining mechanisms (Menlo Park: Benjamin/Cummings)

Busson D., Gans M., Komitopolou K. and Masson M. 1983 Genetic analysis of 3 dominant female-sterile mutations located on the X-chromosome of Drosophila melanogaster. Genetics 105: $309-325$

Chandra H. S. 1986 X chromosomes and dosage compensation. Nature (London) 319:18

Charlesworth B. 1978 Model for evolution of $Y$ chromosomes and dosage compensation. Proc. Natl. Acad. Sci. USA 75: 5618-5622 
Cline T. W. 1986 A female-specific lethal lesion in an X-linked positive regulator of the Drosophila sex determination gene, Sex-lethal. Genetics 113: 641-663

Cock A. G. 1964 Dosage compensation and sex chromatin in non-mammals. Genet. Res. 5: 354-365

Davidson E. H. 1986 Gene activity in early development (New York: Acadenic Press)

Driever W. and Nusslein-Volhard C. 1989 The bicoid protein is a positive regulator of hunchback transcription in the early Drosophila embryo. Nature (London) 337: 138-143

Flach G., Johnson M. H., Braude P. R., Taylor R. A. S. and Bolton V. N. 1982 The transition from maternal to embryonic control in the 2-cell mouse embryo. EMBO J. 1: 681-686

Gartler S. M., Andina R. and Gant N. 1975 Ontogeny of X-chromosome inactivation in the female germ line. Exp. Cell Res. 91: 454-457

Gergen J. P. and Wieschaus E. 1986 Dosage requirements for runt in the segmentation of Drosophila embryos. Cell 45: 289-299

Grula J. W. and Taylor O. R., Jr. 1980 The effect of X-chromosome inheritance on mate selection behaviour in the sulfur butterflies, Colias eurytheme and Colias philodice. Evolution 34: 688-695

Hebbert D. R. 1984 Dosage compensation of the sex-linked enzyme phosphoglucomutase in the Orthoptera. Heredity 53: 361-369

Homyk T., Jr. and Emerson C. P., Jr. 1988 Functional interactions between unlinked muscle genes within haploinsufficient regions of the Drosophila genome. Genetics 119: 105-121

Ingham P. W. 1988 The molecular genetics of embryonic pattern formation in Drosophila. Nature (London) 335: 25-34, 744

John B. and Miklos G. L. G. 1988 The eukaryote genome in development and evolution (London: Allen and Unwin)

Johnson M. S. and Turner J. R. G. 1979 Absence of dosage compensation for a sex-linked enzyme in butterflies (Heliconius). Heredity 43: $71-77$

Kacser H. and Burns J. A. 1981 The molecular basis of dominance. Genetics 97: 639-666

Klingler M., Erdelyi M., Szabad J. and Nussiein-Volhard C. 1988 Function of torso in determining the terminal anlagen of the Drosophila embryo. Nature (London) 335: 275-277

Kongsuwan K., Yu Q., Vincent A., Frisardi M. C., Rosbash M., Lengyel. J. A. and Merriam J. 1985 A Drosophila Minute gene encodes a ribosomal protein. Nature (Lonlon) 317: 555-558

Last R. L., Maddock J. R. and Woolford J. L., Jr. 1987 Evidence for related functions of the RNA genes of Saccharomyces cerevisiae. Genetics 117:619-631

Lindsley D. L., Sandler H., Baker B. S., Carpenter A. T., Denell R. E., Hall J. C., Jacobs P. A., Miklos, G. L. G., Davis, B. K. and Gethmann, R. C. 1972 Segmental aneuploidy and genetic gross structure of the Drosophila genome. Genetics 71: 157-184

Locke J., Kotarski M. A. and Tartof K. D. 1988 Dosage-dependent modifiers of position elfect variegation in Drosophila and a mass action model that explains their effect. Genetics 120: 181-198

Lohs-Scharidin M. 1982 Dicephalic - A Drosophila mutant affecting polarity in follicle organization and embryonic patterning. Wilhelm Roux's Arch. Dev. Biol. 191: 28-36

Lyon M. F. 1961 Gene action in the X-chromosome of the mouse (Mus musculus L.) Nature (London) 190: $372-373$

Lyon M.F. 1974 Mechanisms and evolutionary origins of variable X-chromosome activity in mammals. Proc. R. Soc. Series B 187: 243-268

Maine E. M., Salz H. K., Schedl P. and Cline T. W. 1985 Sex-lethal, a link between sex determination and sexual differentiation in Drosophila melanogaster. Cold Spring Harbor Symp. Quant. Biol. 50: 595604

Mains P. E., Sulston I. A. and Wood W. B. 1990 Dominant maternal-effect mutations causing embryonic lethality in Caenorhabditis elegans. Genetics 125: 351-369

Manseau L. J. and Schupbach T. 1989 The egg came first, of course! Trends Genet. 5: 400-405

Meyer B. J. 1988 Primary events in C. elegans sex determination and dosage compensation. Trends Genet. 4: $337-342$

Mohler J. and Wieschaus E. F. 1986 Dominant maternal-effect mutations of Drosophila melanogaster causing the production of double-abdomen embryos. Genetics 112: 803-822

Muller H. J. 1948 Evidence of the precision of genetic adaptation. Harvey Lect. 43: 165-229

Nusslein-Volhard C., Frohnhofer H. G. and Lehmann R. 1987 Determination of antero-posterior polarity in Drosophila. Science 238: 1675-1681

Ohno S. 1987 Sex chromosomes and sex-linked genes (New York: Springer)

Regan C. L. and Fuller M. T. 1988 Interacting genes that affect microtubule function: the nc2 allele of the haywire locus fails to complement mutations in the testis-specific $\beta$-tubulin gene of Drosophila. Genes Dev. 2: 82-92 
Rice W. R. 1987 Genetic hitchhiking and the evolution of reduced genetic activity of the $Y$ sex chromosome. Genetics 116:161-167

Sargent R. C., Taylor P. D. and Gross M. R. 1987 Parental care and the evolution of egg size in fishes. Am. Nat. 129: 32-46

Schmid M. 1983 Evolution of sex chromosomes and heterogametic systems in Amphibia. Differentiation. 23(Suppl): S13-S22

Sheppard P. M. 1961 Some contributions to population genetics resulting from the study of the Lepidoptera. Adv. Genet. 10: 165-216

Smolik-Utlaut S. M. 1990 Dosage requirements of ullrabithorax and bithoraxoid in the determination of segment identity of Drosophila melanogaster. Genetics 124: 357-366

Stehr G. 1959 Hemolymph polymorphism in a moth and the nature of sex-controlled inheritance. Evolution 13: $537-560$

Strecker T. R., Halsell S. R., Fisher W. W. and Lipshitz H. D. 1989 Reciprocal effect of hyper - and hypoactivity in the Drosophila pattern gene torso. Science 247: 1062-1066

Struhl G. 1983 Role of the esc ${ }^{+}$gene-product in ensuring the selective expression of segment-specific homeotic genes in Drosophila. J. Embryol. Exp. Morph. 76: 297-331

Struhl G., Struhl K. and Macdonald P. M. 1989 The gradient morphogen bicoid is a concentrationdependent transcriptional activator. Cell 57: 1259-1273

Tata J. R. 1986 Coordinated assembly of the developing egg. BioEssays 4: 197-201

Tricoire H. 1988 Dominant maternal interactions with Drosophila segmentation genes. Wilhelm Roux's Arch. Dev. Biol. 197: 115-123

Wilkins A. S. 1986 Genetic analysis of animal development (New York: Wiley)

Wright C. V. E., Cho K. W. Y., Fritz A., Burglin T. R. and DeRobertis E. M. 1987 A Xenopus laevis gene encodes both homeobox-containing and homeobox-less transcripts. EMBO J. 6: 4083-4094 